\title{
Electroweak and supersymmetry breaking from the Higgs boson discovery
}

\author{
Antonio Delgado, ${ }^{1,2}$ Mateo Garcia, ${ }^{3}$ and Mariano Quiros ${ }^{2,4,5}$ \\ ${ }^{1}$ Department of Physics, University of Notre Dame, Notre Dame, Indiana 46556, USA \\ ${ }^{2}$ KITP, University of California, Santa Barbara, California 93106-4030, USA \\ ${ }^{3}$ IFAE, Universitat Autònoma de Barcelona, 08193 Bellaterra, Barcelona, Spain \\ ${ }^{4}$ Department of Physics, CERN-TH Division, CH-1211, Geneva 23, Switzerland \\ ${ }^{5}$ Institució Catalana de Recerca i Estudis Avançats (ICREA) and Institut de Física d'Altes Energìes, UAB, \\ 08193 Bellaterra, Barcelona, Spain
}

(Received 13 May 2014; published 15 July 2014)

\begin{abstract}
We will explore the consequences on the electroweak breaking condition, the mass of supersymmetric partners and the scale at which supersymmetry breaking is transmitted, for arbitrary values of the supersymmetric parameters $\tan \beta$ and the stop mixing $X_{t}$, which follow from the Higgs discovery with a mass $m_{H} \simeq 126 \mathrm{GeV}$ at the LHC. Within the present uncertainty on the top quark mass we deduce that radiative breaking requires $\tan \beta \gtrsim 8$ for maximal mixing $X_{t} \simeq \sqrt{6}$, and $\tan \beta \gtrsim 20$ for small mixing $X_{t} \lesssim 1.8$. The scale at which supersymmetry breaking is transmitted $\mathcal{M}$ can be of order the unification or Planck scale only for large values of $\tan \beta$ and negligible mixing $X_{t} \simeq 0$. On the other hand for maximal mixing and large values of $\tan \beta$ supersymmetry should break at scales as low as $\mathcal{M} \simeq 10^{5} \mathrm{GeV}$. The uncertainty in those predictions stemming from the uncertainty in the top quark mass, i.e. the top Yukawa coupling, is small (large) for large (small) values of $\tan \beta$. In fact for $\tan \beta=1$ the uncertainty on the value of $\mathcal{M}$ is several orders of magnitude.
\end{abstract}

DOI: $10.1103 /$ PhysRevD.90.015016

PACS numbers: 12.60.Jv, 12.60.-i

\section{INTRODUCTION AND SUMMARY}

After the 7 and $8 \mathrm{TeV}$ runs the Large Hadron Collider (LHC) has firmly established the existence of a scalar boson with a mass $m_{H} \simeq 126 \mathrm{GeV}$. In particular the strengths measured in the $W W, Z Z, \gamma \gamma, b \bar{b}$ and $\tau \tau$ decay channels by the ATLAS and CMS collaborations are consistent with the Standard Model (SM) Higgs with a mass $m_{H}=126 \pm 0.4$ (stat) \pm 0.4 (syst) $\mathrm{GeV}$ [1] and $m_{H}=125.3 \pm 0.4$ (stat) \pm 0.5 (syst) GeV [2], respectively. The Higgs discovery is of the utmost importance as it is the first direct experimental confirmation of the mechanism of electroweak (EW) symmetry breaking (EWSB). In the SM it points toward a quartic coupling $\lambda=m_{H}^{2} / v^{2}$, where $v=246 \mathrm{GeV}$, provided by the Higgs potential

$$
V_{\mathrm{SM}}=-m^{2}|H|^{2}+\frac{\lambda}{2}|H|^{4}
$$

where $m^{2}=\lambda v^{2} / 2$, valid at the EW scale $\mathcal{Q}_{\mathrm{EW}}=m_{H}$.

From the theoretical point of view we know that the EW minimum is unstable against quantum corrections (a problem known in the literature as the hierarchy problem) and has to be stabilized by some beyond the SM (BSM) physics, the paradigm of which being supersymmetry and in particular the minimal SM supersymmetric extension (MSSM). Nonetheless another feature of the past LHC runs is that no experimental hints have been found of BSM particles which could stabilize the EW vacuum, but it is putting bounds on the mass of supersymmetric particles [3]. Still, and in view of the forthcoming $\mathrm{LHC}$ run at $13-14 \mathrm{TeV}$, it is interesting to explore the consequences of the present Higgs mass data on a possible underlying supersymmetric theory, in particular on the way supersymmetry triggers EWSB at low energy and on the value of the scale at which supersymmetry is broken.

In this paper we will then consider at face value the present data on the Higgs sector. We will assume that the SM emerges at some scale $\mathcal{Q}_{0}$ from an underlying MSSM, and will extract the relevant information on the mechanism by which the MSSM triggers EWSB and on the scale $\mathcal{M}$ at which supersymmetry is broken in the hidden sector. Consistent with present experimental data we will assume that below the scale $\mathcal{Q}_{0}$ we just have the SM spectrum and the matching conditions are the ones to enforce EWSB at the EW scale $\mathcal{Q}_{\mathrm{EW}}=m_{H}$.

The contents of this paper are as follows. In Sec. II we impose the condition that the SM and the MSSM merge at the scale $\mathcal{Q}_{0}$ and compute it by fixing the Higgs mass in the SM and with fixed values of $\tan \beta$ and the stop mixing $X_{t}$ in the MSSM. We see, not unexpectedly, that for low values of $\tan \beta, \mathcal{Q}_{0}$ is large and insensitive to the mixing $X_{t}$ while for large values of $\tan \beta$ it can be small and sensitive to $X_{t}$. In particular, values of $\mathcal{Q}_{0}$ in the $\mathrm{TeV}$ range require both large values of $\tan \beta(\tan \beta \gtrsim 5)$ and of the mixing $\left(X_{t} \gtrsim 1.8\right)$. Moreover we can translate the condition of EWSB in the $\mathrm{SM}$ to a condition on $m_{2}^{2}\left(\mathcal{Q}_{0}\right)$ (the squared mass of the Higgs doublet that gives a mass to the top quark) and so we can scrutinize on the nature of EWSB, i.e. radiative versus 
nonradiative breaking. ${ }^{1}$ We have found that the nature of EWSB strongly depends on both parameters, $\tan \beta$ and $X_{t}$. In particular we have found that radiative breaking requires $\tan \beta \gtrsim 8$ for maximal mixing and $X_{t} \gtrsim 1.8$ for $\tan \beta \lesssim 20$. Another interesting feature we have found, both in the calculation of the matching scale, i.e. $\mathcal{Q}_{0}$, and the type of EWSB, i.e. the value of $m_{2}^{2}\left(\mathcal{Q}_{0}\right)$, is that the main uncertainty in the calculation comes from the uncertainty in the top quark mass and that it affects mainly low values of $\tan \beta$ (it is mostly insensitive to the actual value of the mixing $X_{t}$ ). In particular for $\tan \beta=1$ and $X_{t}=0$, while for the central value of the top quark mass $\mathcal{Q}_{0} \sim 10^{11} \mathrm{GeV}$, after inserting the $2 \sigma$ uncertainty it can vary in the range $\mathcal{Q}_{0} \in\left[10^{9}, 10^{16}\right] \mathrm{GeV}$ and no sharp prediction can be made. Notice that the results in this section imply precise values of $m_{1}\left(\mathcal{Q}_{0}\right)$ and $m_{2}\left(\mathcal{Q}_{0}\right)$ determined by the EWSB condition and by the condition of decoupling of the heavy Higgs at the matching scale $\mathcal{Q}_{0}$.

In Sec. III we computed the scale at which supersymmetry is transmitted $\mathcal{M}$ by imposing the condition that the MSSM Higgs mass parameters are equal at that scale: $m_{1}(\mathcal{M})=m_{2}(\mathcal{M})$. All of our results in this section are based on this assumption. This one is a natural assumption in most existing models of supersymmetry breaking, including those coming from string theories. Of course, should the condition on $m_{1}(\mathcal{M})$ and $m_{2}(\mathcal{M})$ be changed our results would correspondingly be modified. To compute the value of the scale at which supersymmetry is broken $\mathcal{M}$ we have first followed a bottom-up approach where we assume the ideal conditions that all supersymmetric particles decouple exactly at the scale $\mathcal{Q}_{0}$ (with no thresholds). In the second, top-down, approach we have instead assumed that the supersymmetric parameters are the ones obtained in various models of supersymmetry breaking transmission to the observable sector. In these cases there are different thresholds around the matching scale $\mathcal{Q}_{0}$ but the results are in all cases consistent with the first approach. The main result in the bottom-up approach is (not unexpectedly) that the value of $\mathcal{M}$ depends to a large extent on the supersymmetric parameters $\left[\tan \beta\left(\mathcal{Q}_{0}\right), X_{t}\left(\mathcal{Q}_{0}\right)\right]$. In particular for $X_{t} \geq 0$ large values of $\mathcal{M}$ close to the unification or Planck scale can only be obtained for large values of $\tan \beta$ and small mixing. Of course for those small values of $X_{t}$ the constraint on the Higgs mass imposes large values of $\mathcal{Q}_{0}$, say in the $10-100 \mathrm{TeV}$ region for which the supersymmetric spectrum would be outside the reach of the LHC. On the contrary for large values of $X_{t}$, for instance for maximal mixing, for which $\mathcal{Q}_{0}$ is in the TeV range and the supersymmetric spectrum is inside the reach of the LHC, the scale at which supersymmetry

\footnotetext{
${ }^{1}$ We will conventionally dub radiative breaking the situation where $m^{2}\left(\mathcal{Q}_{0}\right) \leq 0$ although electroweak breaking is triggered in all cases by radiative corrections.
}

breaking is transmitted can go down to the low scales such that gravity mediation mechanisms are precluded. For negative values of $X_{t}$ there is room for large grand unified theory (GUT) or Planckian values of $\mathcal{M}$ provided that $X_{t}$ is in some intermediate region, e.g. $X_{t} \simeq$ -1.5 which can accommodate lower values of $\mathcal{Q}_{0}$ for large values of $\tan \beta$, e.g. $\mathcal{Q}_{0}=\mathcal{O}$ (few) $\mathrm{TeV}$, inside the LHC reach. Again our predictions are affected by the top quark mass uncertainty $\Delta \bar{m}_{t}\left(m_{t}\right)$. As it was the case for the $\mathcal{Q}_{0}$ prediction, the uncertainty affects mainly small values of $\tan \beta$ and it is rather insensitive to the value of $X_{t}$. In the second, top-down, approach we have considered two different cases where supersymmetric parameters unify at the scale $\mathcal{M}$. First we have considered the case of universal soft parameters, by which all squark masses $\left(m_{0}\right)$, all gaugino masses $\left(m_{1 / 2}\right)$ and all Higgs mass parameters $\left(m_{H}\right)$ unify at the scale $\mathcal{M}$. This is a general constrained MSSM where we have separated the Higgs from the sfermion masses and which can appear in gravity mediated supersymmetry breaking theories. We have considered two examples with $\tan \beta=10$ and $X_{t}=0,2$. In agreement with the results of the previous section the case $X_{t}=0$ is consistent with EWSB and the Higgs mass $m_{H}=126 \mathrm{GeV}$ for $\mathcal{M} \simeq 10^{18} \mathrm{GeV}$, while the case $X_{t}=2$ requires supersymmetry breaking at low scale $\mathcal{M} \simeq 10^{6} \mathrm{GeV}$, hard to reconcile with gravity mediation. The second case we have considered is the minimal gauge mediated supersymmetry breaking (GMSB) where the mass of scalars transforming under a gauge group $G_{a}$, with gauge coupling $\alpha_{a}$, and the corresponding gaugino is proportional to $\alpha_{a}(\mathcal{M}) / 4 \pi$ and the trilinear coupling is $A_{t}(\mathcal{M})=0$. Below $\mathcal{M}, A_{t}$ is generated by the MSSM renormalization group equation (RGE) and therefore it gets negative values at the scale $\mathcal{Q}_{0}$, giving then $X_{t}<0$. We have presented two cases with $N=4$ messengers, $\tan \beta=\{15,8\}$ and values of $\mathcal{M}=\left\{10^{8}\right.$, $\left.10^{11}\right\} \mathrm{GeV}$ and $X_{t}=\{-1.8,-1.6\}$ which are consistent with perturbative unification at the MSSM GUT scale. Finally in Sec. IV we present our conclusions.

\section{THE MATCHING AND ELECTROWEAK BREAKING}

The quadratic terms in the MSSM potential can be written as

$$
V_{2}=m_{1}^{2}\left|H_{1}\right|^{2}+m_{2}^{2}\left|H_{2}\right|^{2}+m_{3}^{2}\left(H_{1} \cdot H_{2}+\text { H.c. }\right)
$$

with $H_{1} \cdot H_{2} \equiv H_{1}^{a} \varepsilon_{a b} H_{2}^{b}\left(\varepsilon_{12}=-1\right)$ and we are defining $m_{1}^{2}=m_{H_{1}}^{2}+\mu^{2}$ and $m_{2}^{2}=m_{H_{2}}^{2}+\mu^{2}$, where $m_{H_{i}}$ is the soft breaking mass for $H_{i}$ and $\mu$ is the supersymmetric Higgsino mass. They can also be written as

$$
V_{2}=\left(H_{1}^{\dagger}, \tilde{H}_{2}^{\dagger}\right)\left(\begin{array}{cc}
m_{1}^{2} & m_{3}^{2} \\
m_{3}^{2} & m_{2}^{2}
\end{array}\right)\left(\begin{array}{c}
H_{1} \\
\tilde{H}_{2}
\end{array}\right)
$$


where $\tilde{H}_{2} \equiv \varepsilon H_{2}^{*}$. The diagonalization of the mass matrix

$$
\mathcal{M}_{0}^{2}=\left(\begin{array}{ll}
m_{1}^{2} & m_{3}^{2} \\
m_{3}^{2} & m_{2}^{2}
\end{array}\right)
$$

then yields the mass eigenvalues

$$
m_{\mp}^{2}=\frac{m_{1}^{2}+m_{2}^{2}}{2} \mp \sqrt{\left(\frac{m_{1}^{2}-m_{2}^{2}}{2}\right)^{2}+m_{3}^{4}} .
$$

\section{A. The matching scale}

We wish to match the MSSM with the SM at the (common) scale $\mathcal{Q}_{0} \equiv m_{0}$ of supersymmetric masses. In particular we will rotate the MSSM Higgs sector $\left(H_{1}, \tilde{H}_{2}\right)$ into the basis $(H, \mathcal{H})$ where $H$ is the SM Higgs doublet and $\mathcal{H}$ its heavy orthogonal combination. We then identify the mass squared of the (light) SM Higgs $H$ with the tachyonic mass $m_{-}^{2}=-m^{2}\left(\mathcal{Q}_{0}\right)$ and consequently the mass squared of its (heavy) orthogonal combination $\mathcal{H}$ with $m_{+}^{2} \equiv$ $m_{\mathcal{H}}^{2}=m_{1}^{2}+m_{2}^{2}+m^{2}$. This can be done by the fixing

$$
m_{3}^{4}=\left(m_{1}^{2}+m^{2}\right)\left(m_{2}^{2}+m^{2}\right)
$$

leading to the mixing angle $\beta$ given by

$$
\begin{aligned}
\tan ^{2} \beta & =\frac{m_{1}^{2}+m^{2}}{m_{2}^{2}+m^{2}} \\
\text { i.e. } m^{2} & =\frac{m_{1}^{2}-m_{2}^{2} \tan ^{2} \beta}{\tan ^{2} \beta-1}
\end{aligned}
$$

where all quantities are evaluated at the matching scale $\mathcal{Q}=\mathcal{Q}_{0}$, which rotates the Higgs basis $\left(H_{1}, \tilde{H}_{2}\right)$ into the mass eigenstates $(H, \mathcal{H})$ as

$$
\begin{aligned}
& H=\cos \beta H_{1}-\sin \beta \tilde{H}_{2}, \\
& \mathcal{H}=\sin \beta H_{1}+\cos \beta \tilde{H}_{2} .
\end{aligned}
$$

The potential for the SM Higgs then reads as

$$
V_{\mathrm{SM}}=-m^{2}\left(\mathcal{Q}_{0}\right)|H|^{2}+\frac{\lambda\left(\mathcal{Q}_{0}\right)}{2}|H|^{4}+\cdots .
$$

In order to make a precise calculation of the Higgs mass we have to first match the SM quartic coupling $\lambda$ and the supersymmetric parameters at the scale $\mathcal{Q}_{0}$. We will improve over the tree-level $(\ell=0)$ matching by considering the one-loop $(\ell=1)$ and leading two-loop $(\ell=2)$ threshold effects as given by [4]

$$
\lambda\left(\mathcal{Q}_{0}\right)=\sum_{\ell \geq 0} \Delta^{(\ell)} \lambda
$$

where

$$
\begin{aligned}
\Delta^{(0)} \lambda= & \frac{1}{4}\left(g^{2}+g^{\prime 2}\right) c_{2 \beta}^{2} \\
16 \pi^{2} \Delta^{(1)} \lambda= & 6 y_{t}^{4} s_{\beta}^{4} X_{t}^{2}\left(1-\frac{X_{t}^{2}}{12}\right)-\frac{1}{2} y_{b}^{4} s_{\beta}^{4}\left(\mu / \mathcal{Q}_{0}\right)^{2} \\
& +\frac{3}{4} y_{t}^{2} s_{\beta}^{2}\left(g^{2}+g^{\prime 2}\right) X_{t}^{2} c_{2 \beta} \\
& +\left(\frac{1}{6} c_{2 \beta}^{2}-\frac{3}{4}\right) g^{4}-\frac{1}{2} g^{2} g^{\prime 2}-\frac{1}{4} g^{\prime 4} \\
& -\frac{1}{16}\left(g^{2}+g^{\prime 2}\right)^{2} s_{4 \beta}^{2} \\
\left(16 \pi^{2}\right)^{2} \Delta^{(2)} \lambda= & 16 y_{t}^{4} s_{\beta}^{4} g_{3}^{2}\left(-2 X_{t}+\frac{1}{3} X_{t}^{3}-\frac{1}{12} X_{t}^{4}\right) \\
& +\mathcal{O}\left(h_{t}^{6} s_{\beta}^{4}, g^{4}, g^{2} g^{\prime 2}, g^{\prime 4}\right)
\end{aligned}
$$

and we are using the notation $X_{t}=\left(A_{t}\left(\mathcal{Q}_{0}\right)-\mu\left(\mathcal{Q}_{0}\right) /\right.$ $\tan \beta) / \mathcal{Q}_{0}$, and $s_{\beta} \equiv \sin \beta$ and so on. For the numerical calculation we are also taking into account the $\mathcal{O}\left(y_{t}^{6} s_{\beta}^{4}, \ldots\right)$ two-loop threshold corrections whose explicit expression can be found in Ref. [4]. We are neglecting the corrections proportional to $y_{\tau}^{4}$ as we are not envisaging values of the parameter $\tan \beta$ such that $y_{\tau}$ is relevant.

The couplings $y_{t}$ and $y_{b}$ are the top and bottom Yukawa couplings in the MSSM. They are related to the corresponding SM couplings $h_{t}$ and $h_{b}$ by $[4,5]$

$$
\begin{aligned}
h_{t}= & y_{t} s_{\beta}\left(1-\frac{1}{6 \pi^{2}} g_{3}^{2} \mathcal{Q}_{0}^{2} X_{t} I\left(m_{\tilde{t}_{1}}, m_{\tilde{t}_{2}}, \mathcal{Q}_{0}\right)\right. \\
& \left.+\mathcal{O}\left(y_{b}^{2}, g^{2}, g^{2}\right)\right) \\
h_{b}= & y_{b} c_{\beta}\left(1-\frac{1}{6 \pi^{2}} g_{3}^{2} \mathcal{Q}_{0}^{2} X_{b} I\left(m_{\tilde{b}_{1}}, m_{\tilde{b}_{2}}, \mathcal{Q}_{0}\right)\right. \\
& \left.+\frac{1}{16 \pi^{2}} y_{t}^{2} t_{\beta} \mathcal{Q}_{0}^{2} X_{t} I\left(m_{\tilde{t}_{1}}, m_{\tilde{t}_{2}}, \mathcal{Q}_{0}\right)+\cdots\right)
\end{aligned}
$$

where $X_{b}=\left(A_{t}\left(\mathcal{Q}_{0}\right)-\mu\left(\mathcal{Q}_{0}\right) \tan \beta\right) / \mathcal{Q}_{0}$, we are assuming nearly degenerate spectrum at $\mathcal{Q}_{0}$, and only the leading one-loop QCD and top Yukawa coupling corrections are kept. The function $I(x, y, z)$ can be found in Refs. [4,5].

The parameters of the potential (2.8) have to be run with the SM RGE down to the scale $\mathcal{Q}_{\mathrm{EW}}=m_{H}$, where minimizing the SM potential should lead to $m^{2}\left(m_{H}\right)=$ $\frac{1}{2} m_{H}^{2}, m_{H}^{2}=2 \lambda\left(m_{H}\right) v^{2}$. For a similar analysis see Ref. [6] and especially [7] where the relation between the mass of the Higgs and the scale of supersymmetry breaking was first analyzed. Here in agreement with the used threshold corrections we are using the two-loop RGE as given in [8].

Finally going from the running Higgs mass $m_{H}$ to the pole Higgs mass $M_{H}$ requires the calculation of the Higgs boson self-energy $\Pi\left(p^{2}\right)$ as $M_{H}^{2}=m_{H}^{2}+\Delta \Pi$ where $\Delta \Pi=\Pi\left(p^{2}=M_{H}^{2}\right)-\Pi\left(p^{2}=0\right)$. Here we keep only the 

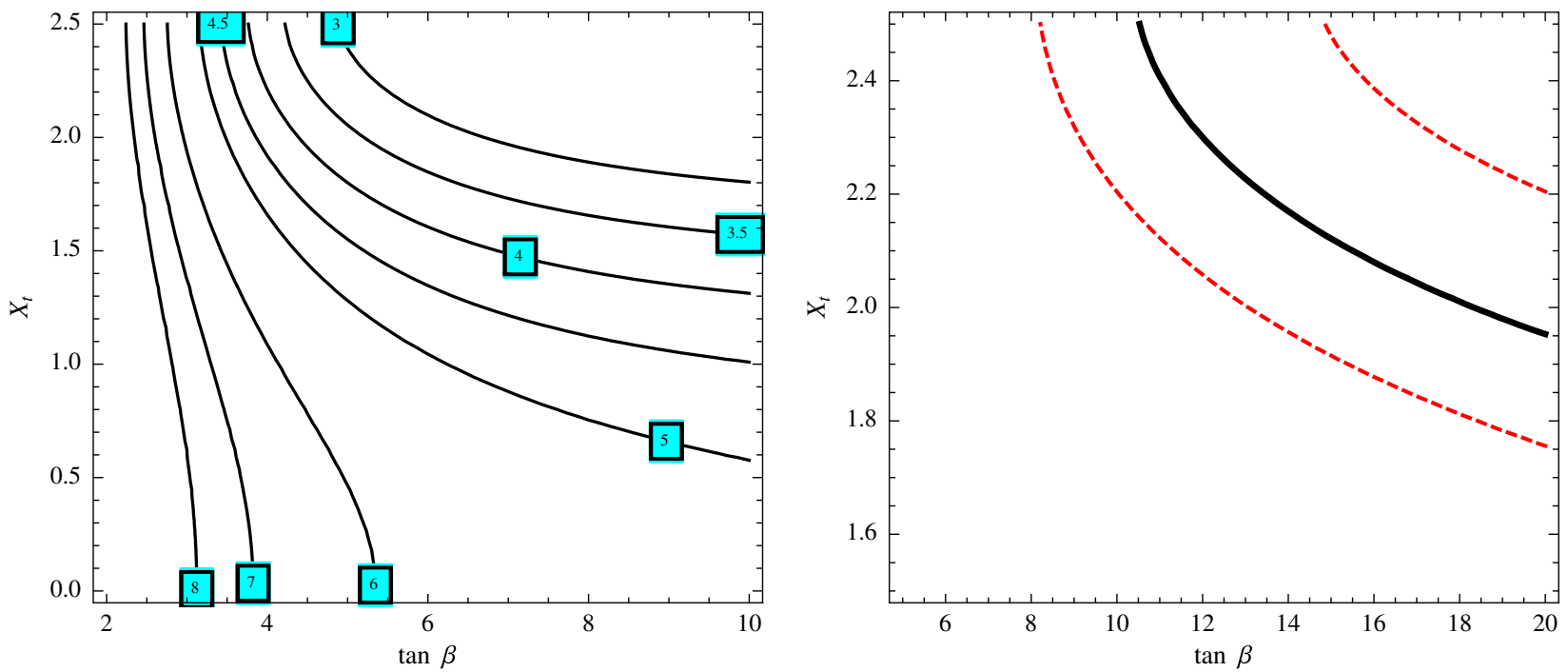

FIG. 1 (color online). Left panel: Contour lines of $\log _{10}\left[\mathcal{Q}_{0} / \mathrm{GeV}\right]$ (for the values specified in the plot) in the plane (tan $\left.\beta, X_{t}\right)$. Right panel: Contour line of $m_{2}^{2}\left(\mathcal{Q}_{0}\right)=0$, as given by Eq. (2.13), in the plane $\left(\tan \beta, X_{t}\right)$. The inner region corresponds to radiative electroweak breaking.

leading correction to $\Delta \Pi$ coming from the top quark loop exchange given by [9]

$$
\begin{aligned}
\Delta \Pi_{t t} & =\frac{3 h_{t}^{2} M_{t}^{2}}{4 \pi^{2}}\left[2-Z\left(M_{t}^{2} / M_{H}^{2}\right)\right], \\
Z(x) & =2 \sqrt{4 x-1} \arctan (1 / \sqrt{4 x-1}), \quad x>1 / 4 .
\end{aligned}
$$

For the actual values of $M_{t} \simeq \bar{m}\left(m_{t}\right)+10 \mathrm{GeV}[10]$ (the pole top quark mass) and $M_{H}$, the correction in (2.12) is of the order of the experimental error in the Higgs mass. Any uncertainty coming from neglected higher order corrections will therefore be much smaller than those from the experimental errors in $\alpha_{3}, M_{t}$ and $M_{H}$, which can be estimated to $\pm 2 \mathrm{GeV}$ at $2 \sigma$ [5].

Notice that, for fixed values of the supersymmetric parameters $\tan \beta$ and $X_{t}, \mathcal{Q}_{0}$ is a function of the Higgs mass $m_{H}$. This prediction comes from the intersection of the function $\lambda(\mathcal{Q})$, which is determined mainly by the value of the Higgs mass [with some dependence on the actual values of $h_{t}\left(m_{H}\right)$ and $\alpha_{3}\left(m_{H}\right)$ ], with the value $\lambda\left(\mathcal{Q}_{0}\right)$ given by Eq. (2.10). So given that the Higgs mass is fixed to $m_{H}=126 \mathrm{GeV}$, we can predict $\mathcal{Q}_{0}=\mathcal{Q}_{0}\left(\tan \beta, X_{t}\right)$ as it is shown in the left panel plot of Fig. 1. We have used as an input the running top mass in the $\overline{\mathrm{MS}}$ scheme evaluated at the top mass $\bar{m}_{t}\left(m_{t}\right)=163.5 \mathrm{GeV}$. We can see that for small values of $\tan \beta$ the values of $\mathcal{Q}_{0}$ are large and insensitive to the values of the mixing $X_{t}$. This is due to the fact that the threshold effect is proportional to $h_{t}^{2}\left(\mathcal{Q}_{0}\right)$ and the Standard Model RGE leads to small values of $h_{t}\left(\mathcal{Q}_{0}\right)$ for large values of the scale $\mathcal{Q}_{0}$. On the other hand for large values of $\tan \beta$ the values of $\mathcal{Q}_{0}$ are smaller and consequently the RGE running is small and $\mathcal{Q}_{0}$ becomes sensitive to the mixing $X_{t}$. In particular values of $\mathcal{Q}_{0}$ in the $\mathrm{TeV}$ region require large values of $\tan \beta(\tan \beta \gtrsim 5)$ and large values of $X_{t}\left(X_{t} \gtrsim 1.8\right)$.

As for the error in $\bar{m}_{t}\left(m_{t}\right)$ it is safe to consider the experimental range of the running top mass to be given by $\Delta \bar{m}_{t}= \pm 2 \mathrm{GeV}$ at $2 \sigma[10,11]$. In order to see the relevance of the error in $\bar{m}_{t}\left(m_{t}\right)$ we plot, in the left panel of Fig. 2, $\mathcal{Q}_{0}$ as a function of $\tan \beta$ for various values of $X_{t}$, and in the right panel of Fig. $2, \mathcal{Q}_{0}$ as a function of $X_{t}$ for different values of $\tan \beta$. In fact the upper border of each band corresponds to $\Delta \bar{m}_{t}=-2 \mathrm{GeV}$ and the lower border to $\Delta \bar{m}_{t}=+2 \mathrm{GeV}$. We can see from both panels of Fig. 2 that the error in the determination of $\mathcal{Q}_{0}$, $\Delta \mathcal{Q}_{0}$ arising from the error in $\bar{m}_{t}\left(m_{t}\right)$ is large (small) for small (large) values of $\tan \beta$. The reason for this behavior is that the error $\bar{m}_{t}\left(m_{t}\right)$ is amplified by the RGE running and it is consequently large (small) for large (small) running, which means small (large) values of $\tan \beta$. In the same way, as we can see from the right panel of Fig. 2, the error $\Delta \mathcal{Q}_{0}$ is uncorrelated with $X_{t}$ as it has little influence on the RGE running. This translates into a big overlapping in the left panel of Fig. 2 for small values of $\tan \beta$ and different values of $X_{t}$. In fact notice that for the limiting case $\tan \beta=1$ and $X_{t}=0$ we have that $\lambda\left(\mathcal{Q}_{0}\right) \lesssim$ 0 and the Standard Model potential is unstable. This corresponds, for the central value of the quark top mass, to $\mathcal{Q}_{0} \sim 10^{11} \mathrm{GeV}$. However for the lowest allowed value of the top quark mass the instability scale can go to Planckian values in agreement with various calculations in the literature $[12,13]$. In this case it has been shown that the Veltman condition [14] (or absence of quadratic divergences) can also be satisfied [15]. 

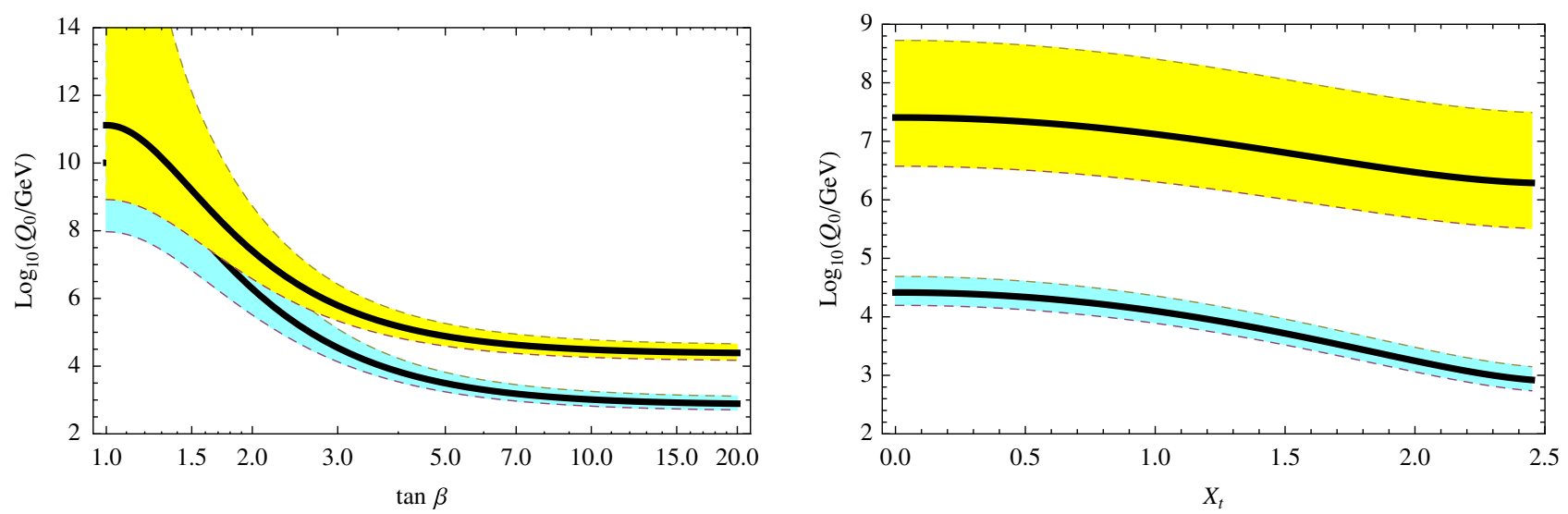

FIG. 2 (color online). Left panel: Plot of $\mathcal{Q}_{0}$ as a function of $\tan \beta$ for $X_{t}=0$ (upper band) and $X_{t}=\sqrt{6}$ (lower band). The width of bands corresponds to the experimental error $\Delta \bar{m}_{t}= \pm 2 \mathrm{GeV}$. Right panel: Plot of $\mathcal{Q}_{0}$ as a function of $X_{t}$ for $\tan \beta=2$ (upper band) and 15 (lower band).

\section{B. Electroweak breaking}

As we have noticed Eq. (2.6) actually implies the existence of the electroweak minimum in the SM effective theory and indeed it is reminiscent of the minimum equation in the MSSM. ${ }^{2}$ In fact Eq. (2.6) can be traded by the SM minimum equation. It can be written as

$$
m_{2}^{2}\left(\mathcal{Q}_{0}\right)=\frac{m_{\mathcal{H}}^{2}\left(\mathcal{Q}_{0}\right)-m^{2}\left(\mathcal{Q}_{0}\right) \tan ^{2} \beta}{\tan ^{2} \beta+1}
$$

where we identify $m_{\mathcal{H}}^{2}\left(\mathcal{Q}_{0}\right) \equiv \mathcal{Q}_{0}^{2}$ and the value obtained for $m_{2}^{2}\left(\mathcal{Q}_{0}\right)$ characterizes the type of electroweak breaking, e.g. radiative versus nonradiative symmetry breaking, ${ }^{3}$ provided that after the SM RGE running we get $m^{2}\left(\mathcal{Q}_{\mathrm{EW}}\right)=m_{H}^{2} / 2$. For instance in the limit $\tan \beta \rightarrow \infty$ [or more precisely for $\tan ^{2} \beta \gg m_{\mathcal{H}}^{2}\left(\mathcal{Q}_{0}\right) / m^{2}\left(\mathcal{Q}_{0}\right)$ ] we get the conditions for radiative breaking, $m_{2}^{2}\left(\mathcal{Q}_{0}\right) \simeq$ $-m^{2}\left(\mathcal{Q}_{0}\right)<0$, while for small values of $\tan \beta$ we get the conditions for nonradiative breaking $m_{2}^{2}\left(\mathcal{Q}_{0}\right) \simeq \frac{m_{\mathcal{Y}}^{2}\left(\mathcal{Q}_{0}\right)}{\tan ^{2} \beta+1}>0$. In particular we show in the right panel of Fig. 1 the contour plot corresponding to $m_{2}^{2}\left(\mathcal{Q}_{0}\right)=0$ for the central value of $\bar{m}_{t}\left(m_{t}\right)$ (thick solid line) and for the $2 \sigma$ values corresponding to $\pm \Delta \bar{m}_{t}\left(m_{t}\right)$ (thin solid lines). The inner area corresponds to the region where there is radiative electroweak symmetry breaking $m_{2}^{2}\left(\mathcal{Q}_{0}\right)<0$ while in the outer region the breaking is not radiative and $m_{2}^{2}\left(\mathcal{Q}_{0}\right)>0$. Of course the values of $m_{2}^{2}\left(\mathcal{Q}_{0}\right)$ should depend to a large extent on the values of $\tan \beta$ and $X_{t}$.

\footnotetext{
${ }^{2}$ Were we neglecting the Standard Model RGE running both equations would be equivalent upon identification of $m_{H}^{2} \leftrightarrow m_{Z}^{2}$.

${ }^{3}$ Although EW breaking is in all cases driven by the MSSM RGE running from $\mathcal{M}$ to $\mathcal{Q}_{0}$, we will be conventionally dubbing radiative breaking the case where $m_{2}^{2}\left(\mathcal{Q}_{0}\right) \leq 0$ so that the EW breaking proceeds by a tachyonic mass as in the SM.
}

In Fig. 3 we plot the absolute value of $m_{2},\left|m_{2}\left(\mathcal{Q}_{0}\right)\right|$, as a function of $\tan \beta$ for different values of $X_{t}$ (left panel) and as a function of $X_{t}$ for different values of $\tan \beta$ (right panel). Notice that points where electroweak breaking becomes radiative are characterized by the fact that $\left|m_{2}\right|=0$ and for larger values of $\tan \beta$ (left panel of Fig. 3) or larger values of $X_{t}$ (right panel of Fig. 3), $m_{2}^{2}$ becomes negative and thus $\left|m_{2}\right|$ takes on positive values. Again we can see that, as for the results in Fig. 2, the effects of the error $\Delta \bar{m}_{t}\left(m_{t}\right)$ are amplified for small values of $\tan \beta$ while they stay small for large values of $\tan \beta$. We can also see that radiative breaking only occurs for large values of $\tan \beta, \tan \beta \gtrsim 8$, and/or large values of the mixing $X_{t} \gtrsim 1.8$ in the range $\tan \beta \lesssim 20$.

\section{Supersymmetry breaking scale}

In the previous section we have computed, using the measured value of the Higgs mass, the value of the scale $\mathcal{Q}_{0}$ at which the MSSM matches with the Standard Model and the value of the parameter $m_{2}^{2}\left(\mathcal{Q}_{0}\right)$ which guarantees a correct electroweak Standard Model breaking at the scale $\mathcal{Q}_{\mathrm{EW}}=m_{H}$. We are here making the conservative assumption (alas, consistent with present experimental data) that only the SM states survive below the matching scale $\mathcal{Q}_{0}$. For large values of $\mathcal{Q}_{0}$ this amounts to assuming a high-scale MSSM beyond $\mathcal{Q}_{0}$, in contradistinction with other possibilities, as those dubbed as split (or minisplit) supersymmetry. Using these tools we will now get information on the scale at which supersymmetry breaking is transmitted $\mathcal{M}$.

As we have seen both $\mathcal{Q}_{0}$ and $m_{2}^{2}\left(\mathcal{Q}_{0}\right)$ are (for fixed values of the Standard Model parameters) functions of the MSSM parameters $\tan \beta$ and $X_{t}$ defined at the scale $\mathcal{Q}_{0}$ : $\mathcal{Q}_{0} \equiv f_{0}\left(\tan \beta, X_{t}\right)$ and $m_{2}^{2}\left(\mathcal{Q}_{0}\right) \equiv f_{2}\left(\tan \beta, X_{t}\right)$. Now from the EWSB condition (2.6) one can also compute $m_{1}^{2}\left(\mathcal{Q}_{0}\right) \equiv$ $f_{1}\left(\tan \beta, X_{t}\right)$ as

$$
m_{1}^{2}\left(\mathcal{Q}_{0}\right)=m_{2}^{2}\left(\mathcal{Q}_{0}\right) \tan ^{2} \beta+m^{2}\left(\mathcal{Q}_{0}\right)\left(\tan ^{2} \beta-1\right)
$$



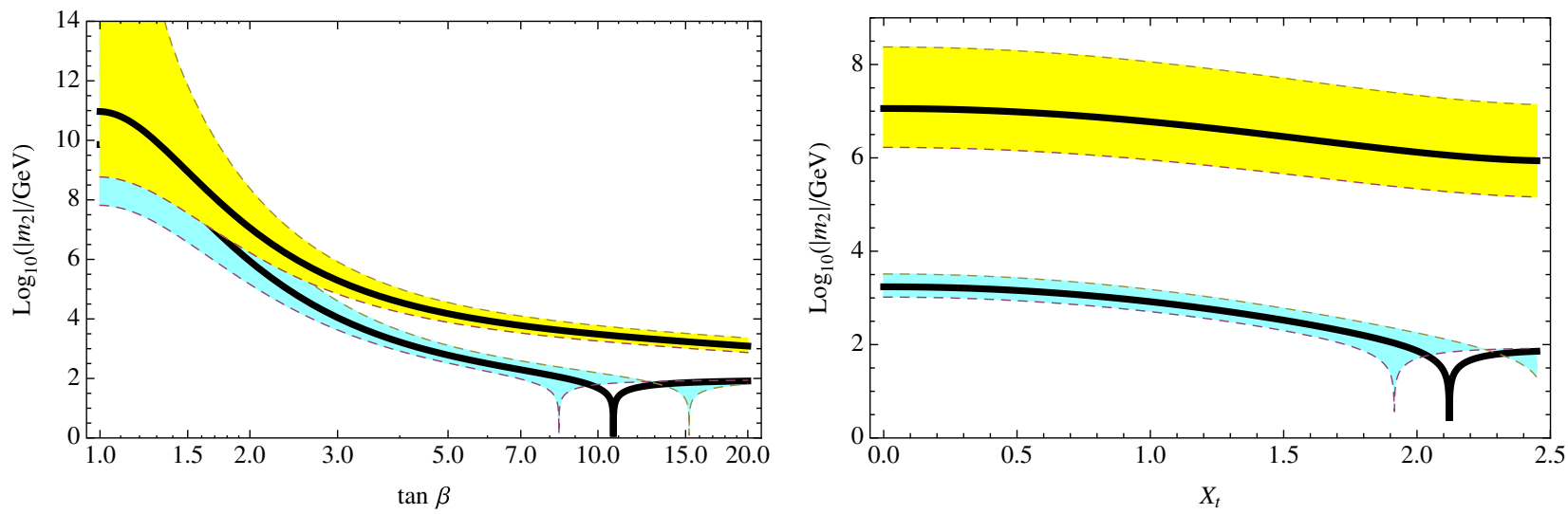

FIG. 3 (color online). Left panel: Plot of $\left|m_{2}\left(\mathcal{Q}_{0}\right)\right|$ as a function of $\tan \beta$ for $X_{t}=0$ (upper band) and $X_{t}=\sqrt{6}$ (lower band). The width of bands corresponds to the experimental error $\Delta \bar{m}_{t}= \pm 2 \mathrm{GeV}$. Right panel: Plot of $\left|m_{2}\left(\mathcal{Q}_{0}\right)\right|$ as a function of $X_{t}$ for tan $\beta=2$ (upper band) and 15 (lower band).

so that both squared mass parameters $m_{1}^{2}$ and $m_{2}^{2}$ are fixed at the scale $\mathcal{Q}_{0}$ for fixed values of $\tan \beta$ and $X_{t}$. We will now define the scale at which supersymmetry is transmitted $\mathcal{M}$ as the scale at which

$$
m_{1}^{2}(\mathcal{M})=m_{2}^{2}(\mathcal{M})
$$

where we are running the MSSM parameters from the scale $\mathcal{Q}=\mathcal{Q}_{0}$ to the scale $\mathcal{Q}=\mathcal{M}$ by using the two-loop RGE [16]. Notice that this condition is rather generic in most models of supersymmetry breaking, as models based on gravity mediation or minimal gauge mediation, as well as in string constructions [17-19].

As we are assuming that the effective theory below $\mathcal{Q}_{0}$ is just the Standard Model we are implicitly assuming that, at the matching scale the heavy Higgs $\mathcal{H}$ decouples, so that $m_{\mathcal{H}}\left(\mathcal{Q}_{0}\right)=\mathcal{Q}_{0}$. On the other hand the scale at which supersymmetry breaking is transmitted, given by (3.2), does have little dependence on the spreading on boundary conditions imposed for the rest of the supersymmetric spectrum. Actually any moderate splitting among the different superpartners will have little impact in the value of $\mathcal{M}$, as its dependence is logarithmic, and the corresponding results fall inside the bands defined by the experimental errors in $M_{t}$ and $\alpha_{3}\left(M_{Z}\right)$, as we have checked in all cases studied in Sec. III B. Thus we will next consider two generic situations.

\section{A. Bottom-up approach}

The most precise (and ideal) way by which the Standard Model will emerge as the low energy effective theory below the matching scale $\mathcal{Q}_{0}$ is when all supersymmetric particles are (approximately) degenerate at the decoupling scale. ${ }^{4}$ So we will here assume for all sfermions $(\tilde{f})$, Higgsinos (with

\footnotetext{
${ }^{4}$ Of course in practice there should be some spreading of supersymmetric masses over the scale $\mathcal{Q}_{0}$, a (more realistic) situation which will be studied in the next section.
}

mass $\mu$ ) and gauginos a degenerate mass at the matching scale $\mathcal{Q}_{0}$

$$
m_{\tilde{f}}\left(\mathcal{Q}_{0}\right)=M_{i}\left(\mathcal{Q}_{0}\right)=\mu\left(\mathcal{Q}_{0}\right)=\mathcal{Q}_{0} \quad(i=1,2,3) .
$$

We will leave $X_{t}\left(\mathcal{Q}_{0}\right)$ [and consequently the mixing $\left.A_{t}\left(\mathcal{Q}_{0}\right)\right]$ and $\tan \beta\left(\mathcal{Q}_{0}\right)$ as free parameters in the plots.

Note that by imposing the matching scheme in Eq. (3.3) the merging between the SM and the MSSM happens at the scale $\mathcal{Q}_{0}$ and the running from the low-scale $\mathcal{Q}_{0}$ to the highscale $\mathcal{M}$ can be done straightforwardly using the two-loop MSSM RGE and the boundary conditions (3.3). This is shown in the left panel (right panel) of Fig. 4 where we plot contour lines of constant $\log _{10}(\mathcal{M} / \mathrm{GeV})$ in the $\left(\tan \beta, X_{t}\right)$ plane for the central value of the top quark mass and positive (negative) values of the parameter $X_{t}$.

We can see from the left panel of Fig. 4 that having supersymmetry breaking transmission at high scale requires both large values of $\tan \beta$ and small and positive values of the mixing $X_{t}$. For example for values of $\mathcal{M}$ of the order of the unification scale $\mathcal{M} \simeq 10^{16} \mathrm{GeV}$ one requires $\tan \beta \gtrsim 3$ and $X_{t} \lesssim 0.3$. Moreover for large values of $\tan \beta$ the value of $\mathcal{M}$ depends almost uniquely on the mixing $X_{t}$. For example even for $\tan \beta \simeq 20$ the scale at which supersymmetry is broken can go down to values as low as $\mathcal{M} \sim 10^{5}-10^{6} \mathrm{GeV}$ for values of the mixing $X_{t} \simeq 2$. On the other hand for low values of $\tan \beta$ and large values of $X_{t}$ there is small dependence on the mixing. As we can see from the left panel of Fig. 4 for values $X_{t} \simeq 0$ we can get values of $\mathcal{M}$ as large as $M_{P}$. For negative values of $X_{t}$ the value of $\mathcal{M}$ grows quickly to trans-Planckian values and rapidly disappears as there is no solution to Eq. (3.2). A solution appears again for values $X_{t} \simeq-1.5$ for which we have again values of $\mathcal{M} \simeq M_{P}$, and again the values of $\mathcal{M}$ decrease when we increase the absolute value of $X_{t}$ as we have shown in the right panel of Fig. 4.

Of course, as it was the case of the matching scale $\mathcal{Q}_{0}$, the scale at which supersymmetry is transmitted $\mathcal{M}$ is 

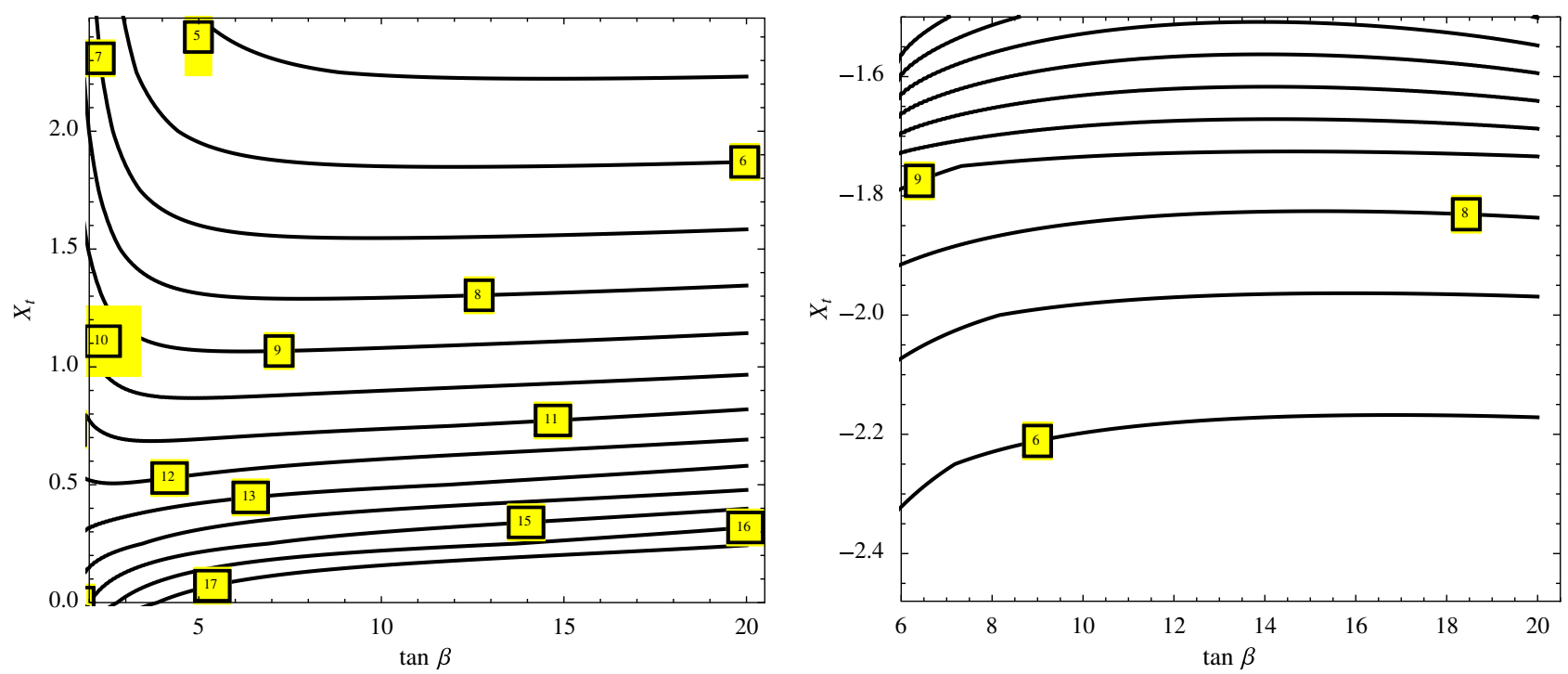

FIG. 4 (color online). Contour lines of constant $\log _{10}[\mathcal{M} / \mathrm{GeV}]$ in the $\left(\tan \beta, X_{t}\right)$ plane for $X_{t} \geq 0$ (left panel) and $X_{t}<0$ (right panel).

affected by the experimental error in the determination of the top quark mass $\Delta \bar{m}_{t}$. This effect is shown numerically in Fig. 5. We plot in the left panel of Fig. $5 \log _{10}(\mathcal{M} / \mathrm{GeV})$ as a function of $\tan \beta$ for different values of the mixing $X_{t}=0,0.5$, and $\sqrt{6}$ for the values of the $\overline{\mathrm{MS}}$ top quark mass $\bar{m}_{t}\left(m_{t}\right)=163.5 \pm 2 \mathrm{GeV}$. This effect is mainly inherited from the uncertainty in the determination of the matching scale $\mathcal{Q}_{0}$, which explains why the effect is larger for $\tan \beta=1$. Similarly the plot of $\log _{10}(\mathcal{M} / \mathrm{GeV})$ as a function of $X_{t}$ for fixed values of $\tan \beta=2$ and 15 is shown in the right panel of Fig. 5 where we can also see that the uncertainty in the determination of $\mathcal{M}$ decreases with increasing values of $\tan \beta$.

\section{B. Top-down approach}

In the previous section we have assumed that all supersymmetric particles exactly decouple at the matching scale

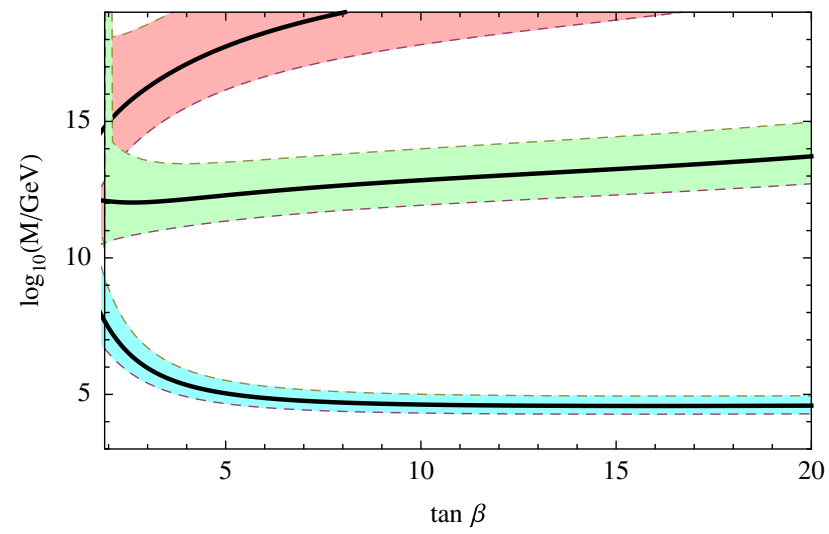

$\mathcal{Q}_{0}$, by which we were assuming a degenerate spectrum at this scale. Of course this is not the generic case in (realistic) models of supersymmetry breaking which provide some pattern of masses at the scale $\mathcal{M}$. These masses run, with the MSSM RGE, from the scale $\mathcal{M}$ to $\mathcal{Q}_{0}$ and thus they decouple at the scale $\sim \mathcal{Q}_{0}$ with different thresholds.

In this section we will consider different supersymmetric spectra, for which the scale at which supersymmetry breaking is transmitted and the matching scale with the Standard Model satisfies the general values which have been obtained in the previous section: in particular they are consistent with electroweak symmetry breaking with a Higgs mass of $126 \mathrm{GeV}$. We will not commit ourselves to any particular mechanism of supersymmetry breaking but instead will consider a generic pattern of supersymmetric spectra at the scale where supersymmetry breaking is transmitted, which can arise from different mechanisms. In particular we will consider two classes of models, which

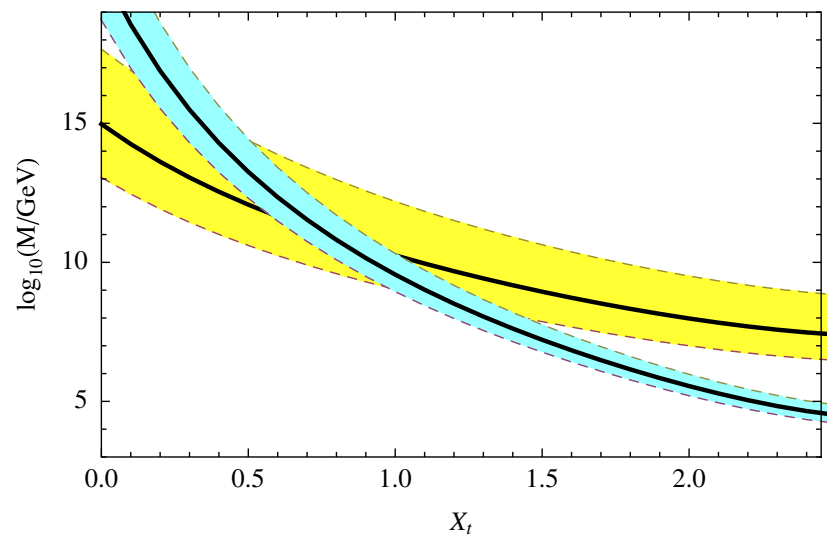

FIG. 5 (color online). Left panel: Plot of $\log _{10}[\mathcal{M} / \mathrm{GeV}]$ as a function of $\tan \beta$ for $X_{t}=0$ (upper band), $X_{t}=0.5$ (central band) and $X_{t}=\sqrt{6}$ (lower band). The width of bands corresponds to the experimental error $\Delta \bar{m}_{t}= \pm 2 \mathrm{GeV}$. Right panel: Plot of $\mathcal{M}$ as a function of $X_{t}$ for $\tan \beta=2$ (wider band) and 15 (narrower band). 

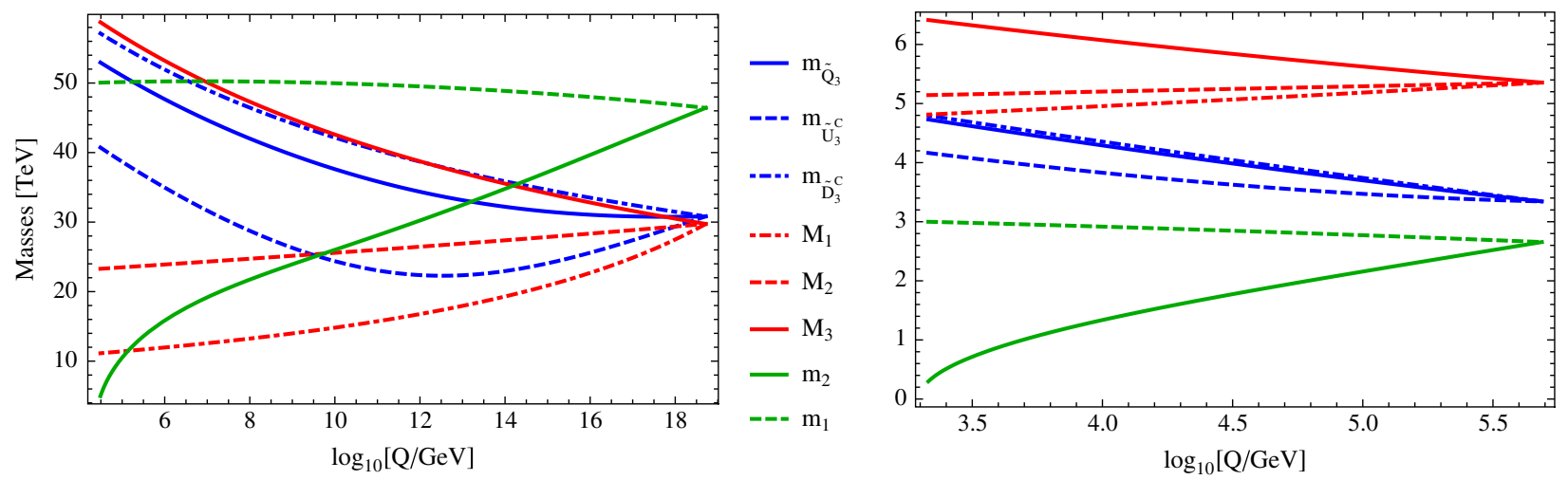

FIG. 6 (color online). RGE running between $\mathcal{M}$ and $\mathcal{Q}_{0}$ of the supersymmetric spectrum for the case $\tan \beta=10, X_{t}=0$ (left panel) and $X_{t}=2$ (right panel) with universal boundary conditions.

are simply particular examples while many others can be easily found and studied:

(i) Models with universal soft parameters, typical of gravity mediatedlike models, although not necessarily arising from gravity mediation.

(ii) Gauge mediated models, where the values of supersymmetric parameters satisfy, at the scale $\mathcal{M}$, typical ratios provided by gauge mediation.

\section{Universal soft parameters}

In this section we are going to consider some universal soft breaking parameters at the scale at which supersymmetry breaking is transmitted $\mathcal{M}$. In particular we will assume the rather general pattern

$$
\begin{aligned}
m_{\tilde{Q}_{3}}(\mathcal{M}) & =m_{\tilde{U}_{3}^{c}}(\mathcal{M})=m_{\tilde{D}_{3}^{c}}(\mathcal{M}) \equiv m_{0}, \\
M_{i}(\mathcal{M}) & \equiv m_{1 / 2}, \quad m_{1}(\mathcal{M})=m_{2}(\mathcal{M})
\end{aligned}
$$

by which all third generation squarks ${ }^{5}$ are degenerate at the scale $\mathcal{M}$, as well as the three gauginos and the two MSSM Higgs doublets. We have then considered the common masses $m_{0}$ and $m_{1 / 2}$ as free parameters only subject to the constraint of getting a correct electroweak symmetry breaking.

We have considered in Fig. 6 two generic models which correspond to $\tan \beta=10$, and $X_{t}=0$ (left panel) and $X_{t}=$ 2 (right panel). As for the case of $X_{t}=0$ a quick glance at the left panel of Fig. 1 shows that the matching scale is $\mathcal{Q}_{0} \sim 100 \mathrm{TeV}$ while from Fig. 4 the scale where supersymmetry breaks is $\mathcal{M} \sim 2 \times 10^{18} \mathrm{GeV}$. Also from the right panel of Fig. $1\left(X_{t}=0\right.$ does not appear in the plot as it is well below the area where radiative breaking exists) we see that the breaking is not radiative in the sense that $m_{2}^{2}\left(\mathcal{Q}_{0}\right)>0$ and indeed from Fig. 3 we can see that,

\footnotetext{
${ }^{5}$ Third generation sleptons as well as first and second generation sfermions do not play any role in the RGE and thus their values decouple from the present problem.
}

according with the correct electroweak symmetry breaking, $m_{2}\left(\mathcal{Q}_{0}\right) \simeq 3 \mathrm{TeV}$. As we can see from the left panel of Fig. 6 the values for the common squark and gaugino masses which fit these conditions are $m_{0} \simeq m_{1 / 2} \simeq$ $30 \mathrm{TeV}$. Also the value of $X_{t}=0$ at the matching scale $\mathcal{Q}_{0}$ translates into the mixing $A_{t}(\mathcal{M}) \simeq 1.7 m_{0}$. Notice that, as the value of $\mathcal{M}$ is around the Planck scale, this scenario could arise in models where supersymmetry breaking is transmitted by gravitational interactions.

If we now increase the value of $X_{t}$, as in the right panel of Fig. 6, in which $X_{t}=2$, then looking again at Fig. 1 we see that the matching scale is $\mathcal{Q}_{0} \sim 1 \mathrm{TeV}$ and the electroweak breaking is (almost) radiative as $m_{2}\left(\mathcal{Q}_{0}\right) \sim 100 \mathrm{GeV}$. Likewise, from Fig. 4, the scale at which supersymmetry is broken is $\mathcal{M} \sim 5 \times 10^{5} \mathrm{GeV}$. Here we can see a general phenomenon by which the scale where supersymmetry breaking is transmitted (i.e. the scale of unification of $m_{1}$ and $m_{2}$ ) strongly goes down when the mixing increases if we fix the correct conditions for electroweak breaking. The reason is the contribution of the mixing to the RGE as

$$
\beta_{m_{2}^{2}}=\frac{3 h_{t}^{2}}{4 \pi^{2}} A_{t}^{2}+\cdots .
$$

To prevent electroweak breaking at high scale $\left(\mathcal{Q} \gg \mathcal{Q}_{0}\right)$ we then let the scale $\mathcal{M}$ go down. For the same reason we need gauginos heavier than squarks as the former ones contribute with negative sign to $\beta_{m_{2}^{2}}$. As we can see in the right panel of Fig. 6 this condition translates into $m_{0} \simeq$ $3.3 \mathrm{TeV}$ and $m_{1 / 2} \simeq 5.3 \mathrm{TeV}$ while at the matching scale $\mathcal{Q}_{0}$ all the supersymmetric spectrum is in the interval 3-6 TeV.

\section{Gauge mediated models}

In this section we will apply the previous results to the particular case in which supersymmetry breaking is transmitted to the observable sector by gauge interactions (GMSB). We will assume in particular the minimal GMSB model whose main features we now summarize. 

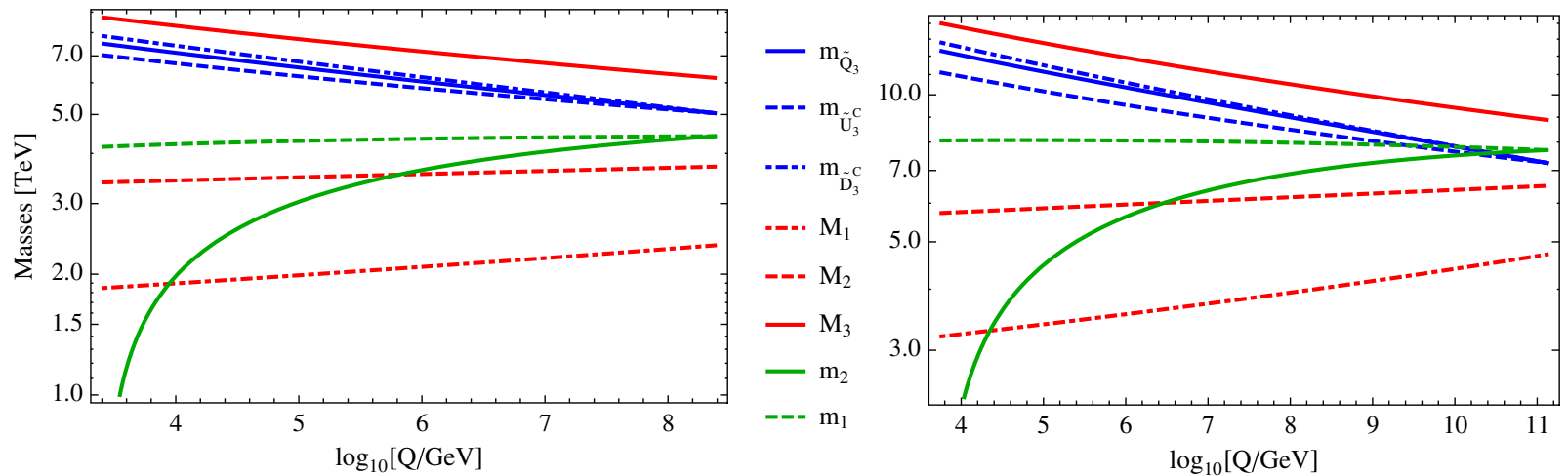

FIG. 7 (color online). RGE running between $\mathcal{M}$ and $\mathcal{Q}_{0}$ of the supersymmetric spectrum for the case $A_{t}(\mathcal{M})=0$ and tan $\beta=15$ (left panel) and $\tan \beta=8$ (right panel) with gauge mediated boundary conditions.

Supersymmetry is broken, in a hidden sector, by a spurion chiral superfield $X=F \theta^{2}$ which is coupled to a set of pairs, $\Phi_{i}+\bar{\Phi}_{i}$, of messenger fields, in vectorlike $\mathbf{r}+$ $\overline{\mathbf{r}}$ representations of the gauge group with the superpotential $W=\sum_{i} \Phi_{i}\left\{\lambda_{i} X+M_{i}\right\} \bar{\Phi}_{i}$.

Gauginos acquire a Majorana mass, by one-loop diagrams given by [20]

$$
M_{a}(\mathcal{M})=\frac{\alpha_{a}(\mathcal{M})}{4 \pi} \Lambda_{G}, \quad \Lambda_{G} \simeq \sum_{i} n_{i} \frac{\lambda_{i} F}{M_{i}}=N \frac{F}{M}
$$

where $n_{i}$ is the Dynkin index for the pair $\Phi_{i}+\bar{\Phi}_{i}{ }^{6}$ and $N=\sum_{i} n_{i}$. For the last equality of Eq. (3.6) we are assuming universal messenger masses as $M_{i} \equiv \lambda_{i} M$ (for $\forall i$ ). Likewise supersymmetric scalars (squarks and sleptons) acquire soft breaking squared masses through two-loop diagrams as

$$
\begin{aligned}
m_{\tilde{f}}^{2}(\mathcal{M}) & =2 \sum_{a} C_{a}^{\tilde{f}} \frac{\alpha_{a}^{2}(\mathcal{M})}{16 \pi^{2}} \Lambda_{S}^{2}, \\
\Lambda_{S}^{2} & =\sum_{i} n_{i} \frac{\left(\lambda_{i} F\right)^{2}}{M_{i}^{2}}=N \frac{F^{2}}{M^{2}}
\end{aligned}
$$

where $C_{a}^{\tilde{f}}$ is the quadratic Casimir of the representation to which $\tilde{f}$ belongs in the group $G_{a}{ }^{7}$, and again for the last equality of Eq. (3.7) we are assuming universal messenger masses. In fact for the case of universal messenger masses the ratio $\Lambda_{G}^{2} / \Lambda_{S}^{2}=N$ is given by the number of messengers, however in more general cases (which can arise e.g. for several $X$ fields overlapping with the Goldstino field)

\footnotetext{
${ }^{6}$ We are using a normalization where $n_{S U(\mathbf{N})}=1$ for the $\mathbf{N}+\overline{\mathbf{N}}$ representation of $S U(\mathbf{N}), n_{U(1)}=6 Y^{2} / 5$, and $\alpha_{1}$ is the $U(1)$ gauge coupling which satisfies the unification condition $\alpha_{a}\left(M_{\mathrm{GUT}}\right)=\alpha_{\mathrm{GUT}}$.

${ }^{7}$ We are using a normalization where for $S U(3)$ triplets, $C_{3}=4 / 3$, for $S U(2)_{L}$ doublets, $C_{2}=3 / 4$, and $C_{1}=3 Y^{2} / 5$. In all cases $C_{a}=0$ for gauge singlets.
}

one can treat $\Lambda_{G}$ and $\Lambda_{S}$ as free parameters. The soft breaking parameter $A_{t}$ is not generated at one loop so we will fix it as $A_{t}(\mathcal{M})=0$ and will let it to develop at the scale $\mathcal{Q}_{0}$ by the MSSM RGE running, which is equivalent to a two-loop effect.

In Fig. 7 we are presenting two typical cases where GMSB is consistent with the conditions imposed by electroweak breaking for a $126 \mathrm{GeV}$ Higgs mass. The case $\tan \beta=15$ is presented in the left panel and $\tan \beta=8$ in the right panel. In both cases we have fixed $\Lambda_{G}=2 \Lambda_{S}$ which corresponds to four messengers, $N=4$, in minimal GMSB models. Both cases are, as we will see, consistent with perturbative unification.

For the case $\tan \beta=15$ in the left panel of Fig. 7 we get $\Lambda_{G} \simeq 1.4 \times 10^{6} \mathrm{GeV}, \mathcal{M} \simeq 3 \times 10^{8} \mathrm{GeV}$, and the scale of supersymmetry breaking $\sqrt{F} \simeq 10^{7} \mathrm{GeV}$ while the expansion parameter $F / \mathcal{M}^{2} \simeq 10^{-3}$ is small, and the gravitino mass is $m_{3 / 2} \simeq 20 \mathrm{keV}$. Notice that $m_{H_{i}}^{2}(\mathcal{M})<m_{\tilde{Q}}^{2}(\mathcal{M})$ although $m_{i}^{2}(\mathcal{M})>m_{\tilde{Q}}^{2}(\mathcal{M})$ because of the contribution of $\mu^{2}$ in $m_{i}^{2}$. This case is perfectly consistent with perturbative unification and the messengers change the value of the gauge couplings at the unification scale by $\delta \alpha_{\mathrm{GUT}}^{-1} \simeq-11$. Even if $A_{t}(\mathcal{M})=0$ a nonzero (and negative) value is generated at the scale $\mathcal{Q}_{0}$ such that $X_{t} \simeq-1.8$.

For the case shown in the right panel of Fig. 7 that corresponds to $\tan \beta=8$ we get the following values of the parameters: $\Lambda_{G} \simeq 2 \times 10^{6} \mathrm{GeV}, \quad \mathcal{M} \simeq 10^{11} \mathrm{GeV}$, $\sqrt{F} \simeq 3 \times 10^{8} \mathrm{GeV}$ with the expansion parameter $F / \mathcal{M}^{2} \simeq 4 \times 10^{-6}$ and $m_{3 / 2} \simeq 20 \mathrm{MeV}$. This case is also consistent with perturbative unification with a value of the gauge couplings at the unification scale and the messengers change the value of the gauge couplings at the unification scale by $\delta \alpha_{\mathrm{GUT}}^{-1} \simeq-8$. Similarly a nonzero negative value of $X_{t}$ is generated as $X_{t} \simeq-1.6$.

\section{CONCLUSIONS}

The Standard Model is consistent with all present experimental data including the recent measurements of 
the Higgs mass and its couplings to gauge bosons and fermions. By the same token experimental data are putting bounds on possible BSM physics whose aim is to solve the SM hierarchy problem, i.e. to understand the hierarchy $\mathcal{Q}_{\mathrm{EW}} / M_{P} \simeq 10^{-16}$, or equivalently the stability of the electroweak vacuum. Even if no hint of new physics has been found by the LHC (and even in the case that no positive signal be found by the future LHC runs) still the stability of the big hierarchy between the LHC energy scale and the Planck scale $\left(\mathcal{Q}_{\mathrm{LHC}} / M_{P} \simeq 10^{-14}\right)$ requires a theoretical explanation, although theories aiming to explain the little hierarchy $\left(\mathcal{Q}_{\mathrm{EW}} / \mathcal{Q}_{\mathrm{LHC}} \simeq 10^{-2}\right)$ do not receive support from the experimental side. On the other hand the paradigm of theories solving the hierarchy problem is supersymmetry, which has roots in superstring theories for which low-scale supersymmetry is not an essential ingredient.

So a possible attitude (that we have adopted in this paper) is to assume that supersymmetry is solving the big hierarchy problem from $\mathcal{Q}_{\mathrm{LHC}} / M_{P}$ (which amounts to a fine-tuning of one part in $10^{28}$ ) but perhaps not necessarily the little hierarchy problem from $\mathcal{Q}_{\mathrm{EW}} / \mathcal{Q}_{\mathrm{LHC}}$ (which amounts to a fine-tuning of around one part in ten thousand) and see what the present data are telling us about the parameters of the supersymmetric theory.

Using then the measured value of the Higgs mass and imposing the conditions for electroweak symmetry breaking we can obtain information on the scale of supersymmetric parameters $\left(\mathcal{Q}_{0}\right)$ and the conditions on how the supersymmetric theory triggers electroweak breaking. Moreover by making the mild assumption that the mass parameters of both Higgs bosons unify at the scale at which supersymmetry breaking is transmitted $\mathcal{M}$ we can obtain rather general information on the latter, as we have described throughout this paper. In models where the former assumption on the Higgs boson mass at $\mathcal{M}$ is not fulfilled the conditions should be accordingly modified.

Our analysis just reflects the present experimental situation concerning the Higgs discovery and the nonobservation of any supersymmetric particle in the LHC7 and LHC8 runs. In the future, when the LHC13-14 run will start in 2015, it might happen that supersymmetric signals are found or that they are not. In both cases the present analysis should be correspondingly constrained. In the case where supersymmetric signals are found, they would give information about our energy scale $\mathcal{Q}_{0}$ which in turn will give indirect information about the scale at which supersymmetry breaking is transmitted $\mathcal{M}$. In the other case, in which supersymmetric signals are not found at the LHC1314 , the data will put a lower bound on the scale $\mathcal{Q}_{0}$ by which also the scale $\mathcal{M}$ will be correspondingly constrained, suggesting that perhaps we will need a higher energy collider to uncover BSM physics as the HE-LHC (at $33 \mathrm{TeV}$ ) and VHE-LHC (at $100 \mathrm{TeV}$ ) [21].

\section{ACKNOWLEDGMENTS}

A. D. and M. Q. would like to thank the National Science Foundation for the partial support under Grant No. PHY1125915. The work of A. D. was partially supported by the National Science Foundation under Grant No. PHY1215979. The work of M.Q. was supported in part by the European Commission under the ERC Advanced Grant No. BSMOXFORD 228169, by the Spanish ConsoliderIngenio 2010 Programme CPAN (Grant No. CSD200700042), and by Grant No. CICYT-FEDER-FPA2011-25948.
[1] G. Aad et al. (ATLAS Collaboration), Phys. Lett. B 716, 1 (2012).

[2] S. Chatrchyan et al. (CMS Collaboration), Phys. Lett. B 716, 30 (2012).

[3] C. Ohm, on behalf of the ATLAS and CMS collaborations, Report No. ATL-PHYS-PROC-2013-299, 2013.

[4] M. S. Carena, J. R. Espinosa, M. Quiros, and C. E. M. Wagner, Phys. Lett. B 355, 209 (1995); S. Heinemeyer, W. Hollik, and G. Weiglein, Phys. Rev. D 58, 091701(R) (1998); , Phys. Lett. B 440, 296 (1998); Eur. Phys. J. C 9 , 343 (1999); J. R. Espinosa and R.-J. Zhang, J. High Energy Phys. 03 (2000) 026; M.S. Carena, H. E. Haber, S. Heinemeyer, W. Hollik, C. E. M. Wagner, and G. Weiglein, Nucl. Phys. B580, 29 (2000); M. S. Carena, J. R. Ellis, A. Pilaftsis, and C.E.M. Wagner, Nucl. Phys. B586, 92 (2000); J. R. Espinosa and R.-J. Zhang, Nucl. Phys. B586, 3 (2000); J. Guasch, W. Hollik, and S. Penaranda, Phys. Lett. B 515, 367 (2001).
[5] P. Draper, G. Lee, and C. E. M. Wagner, Phys. Rev. D 89, 055023 (2014).

[6] G. F. Giudice and R. Rattazzi, Nucl. Phys. B757, 19 (2006).

[7] M. E. Cabrera, J. A. Casas, and A. Delgado, Phys. Rev. Lett. 108, 021802 (2012); G. F. Giudice and A. Strumia, Nucl. Phys. B858, 63 (2012).

[8] H. Arason, D. J. Castano, B. Keszthelyi, S. Mikaelian, E. J. Piard, P. Ramond, and B. D. Wright, Phys. Rev. D 46, 3945 (1992).

[9] J. A. Casas, J. R. Espinosa, M. Quiros, and A. Riotto, Nucl. Phys. B436, 3 (1995); B439, 466(E) (1995).

[10] S. Alekhin, A. Djouadi, and S. Moch, Phys. Lett. B 716, 214 (2012).

[11] I. Masina, Phys. Rev. D 87, 053001 (2013).

[12] G. Degrassi, S. Di Vita, J. Elias-Miro, J. R. Espinosa, G. F. Giudice, G. Isidori, and A. Strumia, J. High Energy Phys. 08 (2012) 098. 
[13] D. Buttazzo, G. Degrassi, P. P. Giardino, G. F. Giudice, F. Sala, A. Salvio, and A. Strumia, J. High Energy Phys. 12 (2013) 089.

[14] M. J. G. Veltman, Acta Phys. Pol. B 12, 437 (1981).

[15] I. Masina and M. Quiros, Phys. Rev. D 88, 093003 (2013).

[16] S. P. Martin and M. T. Vaughn, Phys. Rev. D 50, 2282 (1994); , Phys. Rev. D78, 039903(E) (2008).

[17] L. J. Hall and Y. Nomura, J. High Energy Phys. 03 (2010) 076.
[18] L. E. Ibanez and I. Valenzuela, J. High Energy Phys. 05 (2013) 064.

[19] A. Hebecker, A. K. Knochel, and T. Weigand, Nucl. Phys. B874, 1 (2013).

[20] G. F. Giudice and R. Rattazzi, Phys. Rep. 322, 419 (1999).

[21] M. Koratzinos, A. P. Blondel, R. Aleksan, P. Janot, F. Zimmermann, J. R. Ellis, and M. Zanetti, arXiv:1306.5981. 